\title{
Recherche et dosage de la nitrendipine dans un cas mortel d'intoxication polymédicamenteuse
}

\section{Determination of nitrendipine in a fatal case due to a multidrug poisoning}

\begin{abstract}
Phak-Rop Pos POK ${ }^{(1) *}$, Marie-Noëlle de SAINT-LÉGER ${ }^{(1)}$, Isabelle ELIE ${ }^{(1)}$, Michel MAURAS ${ }^{(1)}$, Joëlle BURLE ${ }^{(1)}$, Erika KUHLMANN(1), Philippe-Emmanuel COIFFAIT ${ }^{(1)}$, Alain VIALA ${ }^{(2)}$
\end{abstract}

(1) Laboratoire de Police Scientifique de Marseille, section Toxicologie (2) Professeur honoraire, Faculté de Pharmacie de Marseille

*Auteur à qui adresser la correspondance : P. Rop P. POK, Laboratoire de Police Scientifique de Marseille, section Toxicologie, 97, Boulevard Camille Flammarion - 13245 Marseille Cedex 4 Tél : 0491628500 - Fax : 0491629770 - E-mail : rop-pos.pok@interieur.gouv.fr

(Reçu le 24 août 2006 ; accepté après modifications le 20 novembre 2006)

\section{RÉSUMÉ}

La nitrendipine est un inhibiteur calcique utilisé dans le traitement de l'hypertension. Nous présentons dans ce travail. une procédure analytique, effectuée en routine au laboratoire, permettant l'analyse toxicologique de la nitrendipine. Nous rapportons ensuite les résultats obtenus dans un cas mortel d'intoxication polymédicamenteuse où la nitrendipine était impliquée. Des échantillons autopsiques de sang, d'urine et de contenu gastrique, prélevés sur la victime ont été analysés par chromatographie en phase gazeuse couplée à la spectrométrie de masse (CPG-SM) et par chromatographie en phase liquide à haute performance avec détecteur à barrette de diodes (CLHP-DBD). Le dosage sanguin de la nitrendipine a été réalisé par CLHP-DBD. La méthode de dosage a été validée et la stabilité du produit a été vérifiée. La dégradation in vitro et in vivo de la nitrendipine a été discutée. La concentration de la nitrendipine dans le sang périphérique $(0,750 \mathrm{mg} / \mathrm{L})$ était 15 fois plus élevée que la valeur. extrême de la fourchette thérapeutique. Les concentrations sanguines des autres composés mis en évidence (paracétamol et triazolam) étaient également toxiques. L'association de ces trois composés était vraisemblablement à l'origine du décès.

MOTS-CLÉS

Nitrendipine, $C P G-S M, C L H P-D B D$, sang.

\section{SUMMARY}

Nitrendipine is a calcium antagonist, used in hypertensive treatment. We present in this work a procedure routinely applied in our laboratory for the toxicological analysis of nitrendipine. Then we reported the result of toxicological analysis from a case of letal poisoning, involying nitrendipine. Necropsic samples from a deceased (blood, urine and gastric content) were analysed by gas chromatography-mass spectrometry (GC-MS) and high performance liquid chromatography-diode-array detection (HPLC-DAD). The quantitation of nitrendipine in blood was carried out by HPLC$D A D$. The quantitative method have been validated and the stability of the drug have been checked. In vitro and in vivo degradation of nitrendipine was discuted. The peripheral. blood concentration of nitrendipine was $0,750 \mathrm{mg} / \mathrm{L}$; this level was 15 times greater than the highest therapeutic level of the drug. The blood concentrations of the associated drugs (paracetamol and triazolam) were within their toxic ranges. The combined toxicity of nitrendipine and associated drugs could be the cause of the death.

\section{KEY-WORDS}

Nitrendipine, GC-MS, HPLC-DAD, blood. 


\section{Introduction}

$\mathrm{La}$ nitrendipine, $\mathrm{C}_{18} \mathrm{H}_{20} \mathrm{~N}_{2} \mathrm{O}_{6}, \mathrm{PM}$ : 360 (figure1) est un inhibiteur calcique appartenant à la famille des dihydropyridines. Elle est utilisée dans le traitement de l'hypertension artérielle. La nitrendipine est commercialisée en France sous la dénomination de Baypress ${ }^{\oplus}$ et Nidrel $^{\oplus}$ $(1,2)$. D'autres spécialités génériques telles que la Nitrendipine Merck $^{\oplus}$ et la Nitrendipine Teva ${ }^{\oplus}$, sont commercialisées. La nitrendipine est sensible à la lumière (2, 3) ; son principal produit de dégradation est la déshydronitrendipine (figure1), qui représente aussi dans l'organisme le métabolite majeur du médicament (4). L'étude de la stabilité des préparations à base de nitrendipine montre qu'il n'y a pratiquement pas de dégradation du principe actif au moins pendant trois ans, si le produit est conservé à l'abri de la lumière et au froid (2).

Par voie orale, la résorption de la nitrendipine est pratiquement totale. Elle se distribue rapidement dans tout l'organisme (1). Elle se lie fortement aux protéines plasmatiques (98\% environ). Sa biotransformation par le foie aboutit à cinq métabolites (2) : le premier, la déshydronitrendipine, est formé par déshydrogénation de la molécule mère $(2,4)$; les quatre autres se forment par hydrolyse, déméthylation et hydroxylation (2-5). Tous ces métabolites sont 1000 fois moins actifs que la nitrendipine (5). L'élimination du principe actif se fait essentiellement par voie urinaire et biliaire et totalement sous forme de métabolites conjugués. La demivie d'élimination varie entre 8 et 23 heures (1). Les concentrations sanguines "thérapeutiques" sont comprises entre 0,010 et $0,050 \mathrm{mg} / \mathrm{L}$ (6). La symptomatologie au cours des surdosages est peu connue $(1,12)$; on peut noter parfois une hypotension sévère avec collapsus, tachycardie ou bradycardie. Plusieurs méthodes ont été proposées pour l'analyse de la nitrendipine dans les milieux biologiques (7-11). Nous rapportons ciaprès le développement de nos méthodes utilisées ainsi que le résultat d'une analyse toxicologique effectuée dans un cas létal d'intoxication où la nitrendipine était impliquée.

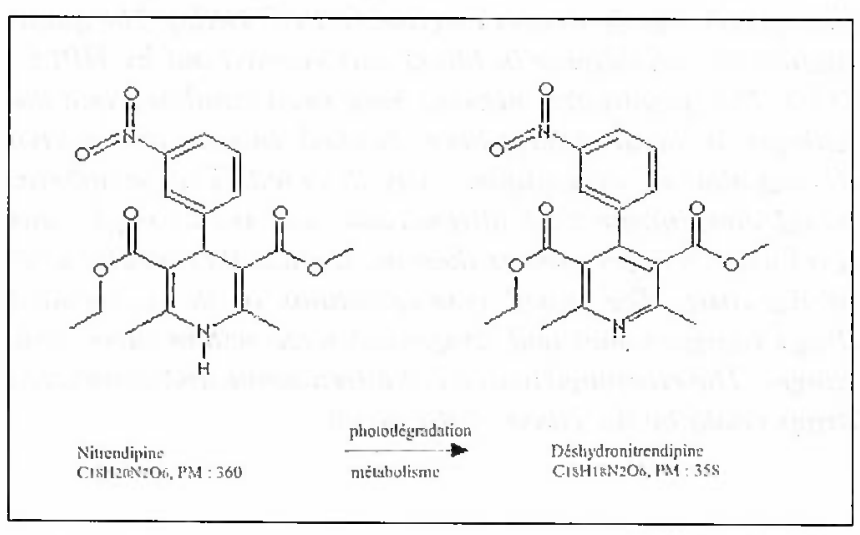

Figure I : Structure chimique de la nitrendipine et de la déshydronitrendipine.

\section{Cas médico-légal}

Il s'agit d'un quinquagénaire retrouvé mort dans sa voiture. Une régurgitation était observée. Aucune trace de violence n'était signalée. L'autopsie a été pratiquée sur la victime. Les échantillons autopsiques de sang périphérique, d'urine et de contenu gastrique feront l'objet d'un examen toxicologique. La nitrendipine a pu être mise en évidence par la procédure analytique appliquée en routine dans notre laboratoire pour la recherche générale des toxiques organiques dans les milieux biologiques, sans avoir modifié les conditions initiales. Le protocole général utilisé comprenait :

- une phase d'extraction par chloroforme/isopropanol en milieu alcalin et acide, par Toxi-tube ${ }^{\oplus} \mathrm{A}$ et Toxitube ${ }^{\circledR} \mathrm{B}$,

- une phase analytique utilisant deux screening successifs par CPG-SM (injection directe et après dérivation) et par CLHP-DBD,

- puis une quantification soit par CPG-SM, soit par CLHP-DBD.

Dans le cas présent, la CLHP-DBD a été choisie pour le dosage de la nitrendipine, car elle a permis de quantifier en même temps les autres composés décelés.

\section{Matériel et méthodes Réactifs et solvants}

Le méthanol, l'acétonitrile et l'eau distillée (qualité CLHP), le chloroforme, le dichlorométhane, l'acide acétique et l'isopropanol (qualité analytique), le phosphate dipotassique et l'acétate d'ammonium (pureté à 99\%) ont été fournis par les laboratoires Carlo Erba (France). Le triméthylchlorosilane (TMS) a été fourni par le laboratoire Macherey Nagel (France). Les Toxitubes $\mathrm{A}$ et $\mathrm{B}$ (kit d'extraction liquide-liquide) ont été fournis par Amilabo (France). La nitrendipine et le clotiazépam (étalon interne) provenaient respectivement des laboratoires Schwarz Pharma (France) et Shire (France). La déshydronitrendipine a été préparée par photodégradation de la nitrendipine, selon la méthode décrite par Tipre (3).

\section{Préparation des solutions étalons}

Les solutions mères de nitrendipine et de clotiazépam à $1 \mathrm{~g} / \mathrm{L}$ dans le méthanol ont été conservées à $-10^{\circ} \mathrm{C}$ et à l'abri de la lumière. Les solutions filles à $0,1 \mathrm{~g} / \mathrm{L}$, $0,01 \mathrm{~g} / \mathrm{L}$ et $0,001 \mathrm{~g} / \mathrm{L}$ ont été préparées extemporanément par dilutions successives de la solution mère avec le même solvant.

La solution de déshydronitrendipine a été préparée à partir de la solution mère de nitrendipine ( $1 \mathrm{~g} / \mathrm{L})$, expo- 
sée à la lumière pendant $70 \mathrm{~h}(10 \mathrm{~h} / \mathrm{jour}$, pendant 7 jours) à $+25^{\circ} \mathrm{C}$. La solution obtenue a été ensuite diluée avec du méthanol afin d'éliminer les autres produits mineurs de dégradation. La dernière solution diluée a servi à l'analyse de la déshydronitrendipine.

\section{Appareillage et conditions chromatogra- phiques}

CPG-SM : La séparation a été effectuée à l'aide d'un chromatographe en phase gazeuse de marque Agilent Technologies, modèle $6890 \mathrm{~N}$, équipé d'une colonne capillaire greffée (5\% phényl-95\% méthyl siloxane ; $15 \mathrm{~m} \times 0,25 \mathrm{~mm}$ d.i ; 0,25 $\mu \mathrm{m}$ d'épaisseur de film) de marque Varian, utilisant de l'hélium comme gaz vecteur. La détection a été assurée par un spectromètre de masse de marque Agilent Technologies, modèle 5973N. L'ionisation des molécules par impact électronique a été faite à $70 \mathrm{eV}$ pour une gamme de masse $\mathrm{m} / \mathrm{z}$ de 40 à 800 . Le débit d'hélium était de $1,5 \mathrm{~mL} / \mathrm{min}$. La température du four était programmée à $50^{\circ} \mathrm{C}$ pendant $1 \mathrm{~min}$, puis de $25^{\circ} \mathrm{C} / \mathrm{min}$ jusqu'à $220^{\circ} \mathrm{C}, 5^{\circ} \mathrm{C} / \mathrm{min}$ jusqu'à $260^{\circ} \mathrm{C}, 30^{\circ} \mathrm{C} / \mathrm{min}$ jusqu'à $330^{\circ} \mathrm{C}$ et maintenue pendant 10 min à ce niveau. Les températures de l'injecteur et de l'interface étaient réglées à $250^{\circ} \mathrm{C}$ et $300^{\circ} \mathrm{C}$ respectivement. Les spectres de masse des composés obtenus ont été comparés à ceux existant dans la bibliothèque NIST fournie avec l'appareillage utilisé. L'ensemble était piloté par un micro-ordinateur équipé du logiciel MSDCHEM ${ }^{\circledR}$ pour Windows XP.

CLHP-DBD : La séparation a été réalisée à l'aide d'un chromatographe en phase liquide Waters, composé d'un module de séparation 2695 réunissant une pompe quaternaire, un dégazeur, un injecteur automatique et un four à colonne et équipé d'une colonne Symmetry C8 Waters ( $25 \mathrm{~cm} \times 4,6 \mathrm{~mm}$ d.i ; particules de $5 \mu \mathrm{m})$. La détection a été assurée par un détecteur à barrette de diodes 996 Waters. Le four à colonne était réglé à $30^{\circ} \mathrm{C}$. La phase mobile était constituée d'un mélange ternaire : solution d'acétate d'ammonium $0,1 \mathrm{M}$ - acétonitrile - méthanol $(\mathrm{v} / \mathrm{v} / \mathrm{v})$. On a utilisé un gradient d'élution à débit constant $(1 \mathrm{~mL} / \mathrm{min})$ pendant $54 \mathrm{~min}$. La composition initiale (phase aqueuse 60\% - acétonitrile 20\% - méthanol 20\%) a été maintenue pendant 2 min. A partir de la $2^{\text {eme }}$ minute, le pourcentage de la phase aqueuse (60\%) a été diminué en trois segments linéaires; le premier pendant $20 \mathrm{~min}$ pour descendre à $40 \%$, le deuxième pendant $16 \mathrm{~min}$ pour descendre à $30 \%$ et le troisième pendant $12 \mathrm{~min}$ pour descendre à $10 \%$. A partir de la $50^{\text {sme }}$ minute, le pourcentage de la phase aqueuse a été remonté linéairement pendant 4 min pour retrouver la composition initiale. Les chromatogrammes et les spectres d'absorption UV-Visible des substances analysées ont été explorés entre 200 et
$400 \mathrm{~nm}$. Les spectres obtenus ont été comparés à ceux des 400 produits de la bibliothèque spectrale du laboratoire Tox222-LPSM, créée dans les conditions décrites ci-dessus. Le dosage a été réalisé à $237 \mathrm{~nm}$. Toutes les données analytiques ont été intégrées par un logiciel Millenium 3.2 Waters.

Screening toxicologique par CPG-SM et CLHP-DBD:

Pour l'analyse par CPG-SM, les échantillons (sang, urine et contenu gastrique) ont été extraits par un mélange de solvants organiques : chloroforme/isopropanol (95: 5, v/v), en milieu alcalin et en milieu acide. La prise d'essai $(1 \mathrm{~mL})$ a été prélevée dans un tube à extraction, puis alcalinisée à $\mathrm{pH} 8,4$ avec la solution de phosphate dipotassique $1 \mathrm{M}(1 \mathrm{~mL})$ ou acidifiée à $\mathrm{pH} 3$ avec l'acide acétique $10 \%(1 \mathrm{~mL})$. Le mélange chloroforme/isopropanol ( $3 \mathrm{~mL}$ ) a été ajouté dans le tube. Après agitation ( $20 \mathrm{~min}$ ) et centrifugation ( $3 \mathrm{~min}$, à 3000 tours $/ \mathrm{min}$ ), la phase organique a été transférée dans un tube conique et évaporée à sec sous courant d'azote. Pour l'analyse en direct, le résidu sec obtenu a été repris par le dichlorométhane $(100 \mu \mathrm{L})$ et une partie aliquote de cette solution $(1 \mu \mathrm{L})$ a été injectée directement dans le chromatographe. Pour l'analyse après dérivation, le résidu a été repris par le triméthylchlorosilane TMS $(100 \mu \mathrm{L})$ et chauffé pendant $20 \min$ à $70^{\circ} \mathrm{C}$ dans un tube fermé. Après refroidissement, une partie aliquote de cette solution $(1 \mu \mathrm{L})$ a été injectée dans le chromatographe. L'analyse de l'échantillon non dérivé apparaît suffisante pour mettre en évidence la présence de nitrendipine et de son métabolite.

Pour l'analyse par CLHP, les échantillons à analyser (sang, urine et contenu gastrique) ont été extraits en milieu alcalin par le Toxi-tube A et en milieu acide par le Toxi-tube B. L'échantillon ( $1 \mathrm{~mL})$ a été ajouté dans le Toxi-tube et complété au trait de jauge du tube par de l'eau distillée. Après agitation manuelle $(3 \mathrm{~min})$ et centrifugation ( $3 \mathrm{~min}$ à 3000 tours $/ \mathrm{min}$ ), la phase organique a été transférée dans un tube Eppendorf conique et évaporée à sec sous courant d'azote. Le résidu sec obtenu a été repris par le méthanol $(100 \mu \mathrm{L})$ et une partie aliquote de cette solution $(20 \mu \mathrm{L})$ a été injectée dans le chromatographe.

Dosage de la nitrendipine dans le sang par CLHP-DBD :

Le dosage de la nitrendipine dans le sang a été réalisé par la méthode CLHP-DBD après extraction par Toxitube $\mathrm{A}$, en présence de clotiazépam comme étalon interne. Le sang périphérique ( $1 \mathrm{~mL}$ ) et la solution de clotiazépam à $0,1 \mathrm{~g} / \mathrm{L}(10 \mu \mathrm{L})$ ont été ajoutés dans le Toxi-tube A. Le mélange a été complété au trait de jauge du Toxi-tube par de l'eau distillée. Après agitation manuelle ( $3 \mathrm{~min}$ ) et centrifugation $(3 \mathrm{~min}$ à 3000 tours / $\mathrm{min}$ ), la phase organique a été transférée dans un tube Eppendorf conique et évaporée à sec sous courant 
d'azote. Le résidu sec obtenu a été repris par le méthanol $(100 \mu \mathrm{L})$; une partie aliquote de cette solution $(20 \mu \mathrm{L})$ a été injectée dans le chromatographe.

\section{Validation de la méthode de dosage}

La méthode de dosage sanguin par CLHP-DBD a été validée par des séries d'essais expérimentaux.

- La courbe d'étalonnage a été réalisée à partir d'échantillons sanguins blancs surchargés de nitrendipine à des concentrations croissantes $(0,020-0,050-0,125$ $0,250-0,500$ et $1 \mathrm{mg} / \mathrm{L}, \mathrm{n}=3$ pour chaque concentration) et extraits dans les mêmes conditions que le sang analysé. La méthode est linéaire pour des concentrations en nitrendipine allant de 0,020 à $1 \mathrm{mg} / \mathrm{L}$ avec un coefficient de corrélation $r^{2}=0,999$ et la droite d'équation suivante : $y=1,627 x-0,0207$ où $\mathrm{y}=$ rapport (en aire) nitrendipine/étalon interne et $\mathrm{x}=$ concentration en $\mathrm{mg} / \mathrm{L}$.

- Les coefficients de variation obtenus après les essais de répétabilité intra-journée $(\mathrm{n}=6)$ et de reproductibilité inter-journée $(\mathrm{n}=6)$ à des concentrations de $0,250 \mathrm{mg} / \mathrm{L}$, $0,500 \mathrm{mg} / \mathrm{L}$ et $1 \mathrm{mg} / \mathrm{L}$, sont inférieurs à $10 \%$.

- Le test d'extraction a été réalisé selon le procédé préconisé sur le sang blanc $(1 \mathrm{~mL})$ surchargé d'une quantité connue de nitrendipine : $0,250 \mu \mathrm{g}, 0,500 \mu \mathrm{g}$ et $1 \mu \mathrm{g}$ ( $n=3$ pour chaque concentration sanguine). Le rapport $(\mathrm{Re})$ en aire (nitrendipine/étalon interne) obtenu dans cette série d'essais a été comparé avec celui obtenu dans la série de témoins (Rt). Pour la série témoin, la nitrendipine est ajoutée à la fin de l'extraction; dans la phase organique avant l'évaporation sous azote. Le pourcentage d'extraction ( $\mathrm{r} \%$ ) est calculé selon la formule $\mathrm{r} \%=(\mathrm{Re}) /(\mathrm{Rt}) \times 100$. Dans l'ensemble, $\mathrm{r} \%$ est supérieur à $60 \%$.

- La limite de détection déterminée à $237 \mathrm{~nm}$ est estimée à $3 \mathrm{ng}$ de nitrendipine (signal/bruit de fond $=3$ ) ; il s'agit d'une limite de détection absolue donnée par le détecteur (à $237 \mathrm{~nm}$ ) après injection de $20 \mu \mathrm{L}$ de la solution (à $0,15 \mathrm{mg} / \mathrm{L}$ ) du produit dans le méthanol. La limite de quantification dans les conditions décrites, à la même longueur d'onde, est estimée à $20 \mathrm{ng}$ par $\mathrm{mL}$ de sang analysé (soit 0,020 mg/L).

- En ce qui concerne la sélectivité de la méthode vis à vis des composés testés (400 composés enregistrés dans la bibliothèque spectrale précitée), quelques interférences peuvent se remarquer, notamment de la part de : la vinburnine (28,81 min), le dihydrocristine mésylate $(28,86 \mathrm{~min})$ et le dihydroergocristine mésylate (28,77 $\mathrm{min})$, dont les temps d'élution sont très proches de celui de la nitrendipine (28,84 min). Aucune inter- férence des constituants endogènes n'a été observée avec la nitrendipine ni avec l'étalon interne. Selon Kann et al. (4) le métabolite déshydrogéné de la nitrendipine, $\mathrm{C}_{18} \mathrm{H}_{18} \mathrm{~N}_{2} \mathrm{O}_{6}, \mathrm{PM}: 358$ (figure1) peut être également retrouvé dans le sang. La sélectivité de la méthode vis à vis de ce métabolite nous a conduit à analyser la déshydronitrendipine, obtenue après la photodégradation de la nitrendipine, ainsi que celle obtenue in vivo. Dans les conditions analytiques proposées, la déshydronitrendipine a été bien séparée de la nitrendipine et caractérisée par son temps de rétention 28,26 min (figure 3a) et son spectre UV-Visible dont le maximum d'absorption est à $265 \mathrm{~nm}$ (figure $3 \mathrm{c}$ ). Cette bonne spécificité est également assurée par la CPG-SM.

\section{Étude de la stabilité de la nitrendipine}

La stabilité in vitro de la nitrendipine a été étudiée selon la procédure décrite par Tipre (3). Pour la stabilité des solutions étalons méthanoliques, le test a été effectué sur la solution à $1 \mathrm{~g} / \mathrm{L}$ conservée dans des conditions différentes (Tableau I). A l'issue des conservations, les solutions filles à $1 \mathrm{mg} / \mathrm{L}$ ont été analysées $(n=3)$. Les résultats obtenus (moyenne des aires du pic de la nitrendipine) ont été comparés à ceux obtenus avant la conservation. Les résultats sont répertoriés dans le Tableau I. Sous l'action de la lumière, la nitrendipine est oxydée en plusieurs composés, dont la déshydronitrendipine qui représente le produit de dégradation le plus important (3). En solution méthanolique à $+4^{\circ} \mathrm{C}$ et à l'abri de la lumière, aucune dégradation de la nitrendipine n'a été observée pendant au moins un mois. En solution méthanolique exposée à la lumière et à la température du laboratoire $\left(+25^{\circ} \mathrm{C}\right)$, la nitrendipine n'est stable que pendant $8 \mathrm{~h}$; après $12 \mathrm{~h}$ d'exposition, la perte en produit initial est de 1,64\% ; elle peut atteindre $51 \%$ au bout de $70 \mathrm{~h}$. Aucune dégradation de la nitrendipine n'a été observée pendant l'extraction et l'analyse. Pour la stabilité du produit dans les échantillons sanguins, le test a été effectué sur les échantillons de sang à $0,250,0,500$ et $1 \mathrm{mg} / \mathrm{L}(\mathrm{n}=3$ pour chaque concentration) conservés à $-10^{\circ} \mathrm{C}$ à l'abri de la lumière pendant une période d'un mois. Après cette période de conservation, les échantillons sanguins ont été analysés selon la procédure décrite. Les résultats obtenus (moyennes des concentrations trouvées) ont été comparés à ceux obtenus avant la conservation. Les résultats sont également répertoriés dans le Tableau I. La nitrendipine est stable pendant au moins un mois. 
Tableau I : Test de stabilité de la nitrendipine.

\begin{tabular}{|c|c|c|}
\hline Conditions de conservation & Temps d'essai & Perte \% \\
\hline Solution méthanolique $+4^{\circ} \mathrm{C}$ à l'abri de la lumière & 0 & 0 \\
& 7 jours & 0 \\
& 15 jours & 0 \\
\hline Solution méthanolique exposée à la lumière et température ambiante du laboratoire $\left(+25^{\circ} \mathrm{C}\right)$ & 30 jours & 0 \\
\hline & $12 \mathrm{~h}$ & 0 \\
& $40 \mathrm{~h}(4 \text { jours })^{*}$ & $22 \%$ \\
\hline Extraction et Analyse & $70 \mathrm{~h}(7 \text { jours })^{*}$ & $51 \%$ \\
\hline Échantillon sanguin $-10^{\circ} \mathrm{C}$ à l'abri de la lumière & $1,5 \mathrm{~h} \mathrm{environ}$ & 0 \\
\hline
\end{tabular}

: : Exposition à la température du laboratoire et seulement $10 \mathrm{~h} /$ jour à la lumière (de $8 \mathrm{~h}$ à $18 \mathrm{~h}$ ).

\section{Résultats et discussion}

Les résultats concernant les paramètres de validation ont été satisfaisants. La nitrendipine a pu être dosée spécifiquement dans le sang. Précisons que la CPG-SM utilisée pour le screening a permis également d'obtenir une séparation parfaite entre la nitrendipine et la déshydronitrendipine.

Le screening toxicologique a permis de mettre en évidence dans le sang périphérique, le contenu gastrique et l'urine, la présence de nitrendipine (associée ou non à son métabolite principal), de paracétamol et de triazolam.

La figure 2 illustre les résultats obtenus après analyse du sang par CPG-SM. Sur le chromatogramme de la figure $2 \mathrm{a}$, le pic n² à 19,60 min a pu être attribué, grâce à son spectre de masse (ion parent $\mathrm{m} / \mathrm{z} 360$; ions fils $m / z 238,210$ ), à la nitrendipine (figure $2 b$ ). Le pic $\mathrm{n}^{\circ} 1$ de la figure $2 \mathrm{a}$, à $15,94 \mathrm{~min}$ (ion parent $\mathrm{m} / \mathrm{z} 358$, ion fils $\mathrm{m} / \mathrm{z} 341,313$ ) a pu être attribué à la déshydronitrendipine (figure $2 \mathrm{c}$ ). La figure 3 illustre les résultats obtenus après analyse du sang par CLHP-DBD. Sur le chromatogramme de la figure $3 a$, le pic portant le $n^{\circ} 4$, à $28,84 \mathrm{~min}$, a pu être attribué, grâce à son spectre UVVisible ( $\max : 237 \mathrm{~nm}$ et $355 \mathrm{~nm}$, min : $305 \mathrm{~nm}$ ), à la nitrendipine (figure $3 b$ ). Le pic $n^{\circ} 3$ de la figure $3 a$, à 28,26 min dont le maximum d'absorption est à $265 \mathrm{~nm}$ a pu être attribué à la déshydronitrendipine (figure 3c). Ce métabolite, pharmacologiquement inactif et vraisemblablement peu formé (pic $n^{\circ} 3$, figure $3 a$ ), n'a pas été quantifié. Les autres composés décelés dans le sang, à savoir, le paracétamol et le triazolam, ont été quant à eux dosés par la méthode CLHP-DBD; les résultats quantitatifs sont résumés dans le Tableau II.

Les inhibiteurs calciques ont plusieurs effets sur le système cardio-vasculaire, sur les fibres lisses et sur le système cérébral. La nitrendipine exerce son effet prédominant sur le système cardio-vasculaire, avec des chutes de tension entraînant en cas de surdosage des complications parfois sérieuses $(1,12)$. D'après la littérature, les concentrations sanguines thérapeutiques de la nitrendipine varient entre 0,010 et $0,050 \mathrm{mg} / \mathrm{L}$ (6). Dans le cas présent, la concentration de la nitrendipine $(0,750 \mathrm{mg} / \mathrm{L})$ dans le sang périphérique était 15 fois plus élevée que la valeur extrême de la fourchette thérapeutique. Ce résultat pourrait témoigner d'un surdosage de nitrendipine. Les concentrations sanguines toxiques et mortelles de la nitrendipine sont encore mal connues. Concernant le paracétamol et le triazolam, les concentrations sanguines mesurées étaient respectivement de $124 \mathrm{mg} / \mathrm{L}$ et $0,047 \mathrm{mg} / \mathrm{L}$. Elles se situaient dans l'intervalle des concentrations considérées toxiques $(13,6)$. Le Tableau II résume ces résultats. Précisons qu'il n'a pas été retrouvé d'alcool éthylique dans le sang et l'urine. Notons encore que, d'après Dolin et al. (14), la nitrendipine peut potentialiser l'effet anesthésique de certaines benzodiazépines. De plus, des interactions d'ordre pharmacocinétique sont susceptibles d'augmenter les concentrations sanguines des toxiques : d'une part, l'association du triazolam, benzodiazépine très sédative, et d'inhibiteurs calciques, tels que le diltiazem et le vérapamil, est formellement déconseillée car elle peut induire une augmentation des concentrations de l'anxiolytique (15); d'autre part, le paracétamol présent à une concentration sanguine toxique $(124 \mathrm{mg} / \mathrm{L})$ était susceptible d'engendrer une insuffisance hépatique et une atteinte rénale, conduisant à une augmentation des concentrations sanguines des autres composés associés. Il est donc possible d'envisager que le paracétamol ait pu être responsable d'une modification de la biotransformation et de l'élimination des substances associées. De ce fait, ces substances se sont accumulées dans le milieu sanguin pour atteindre des concentrations toxiques à l'origine du décès. 


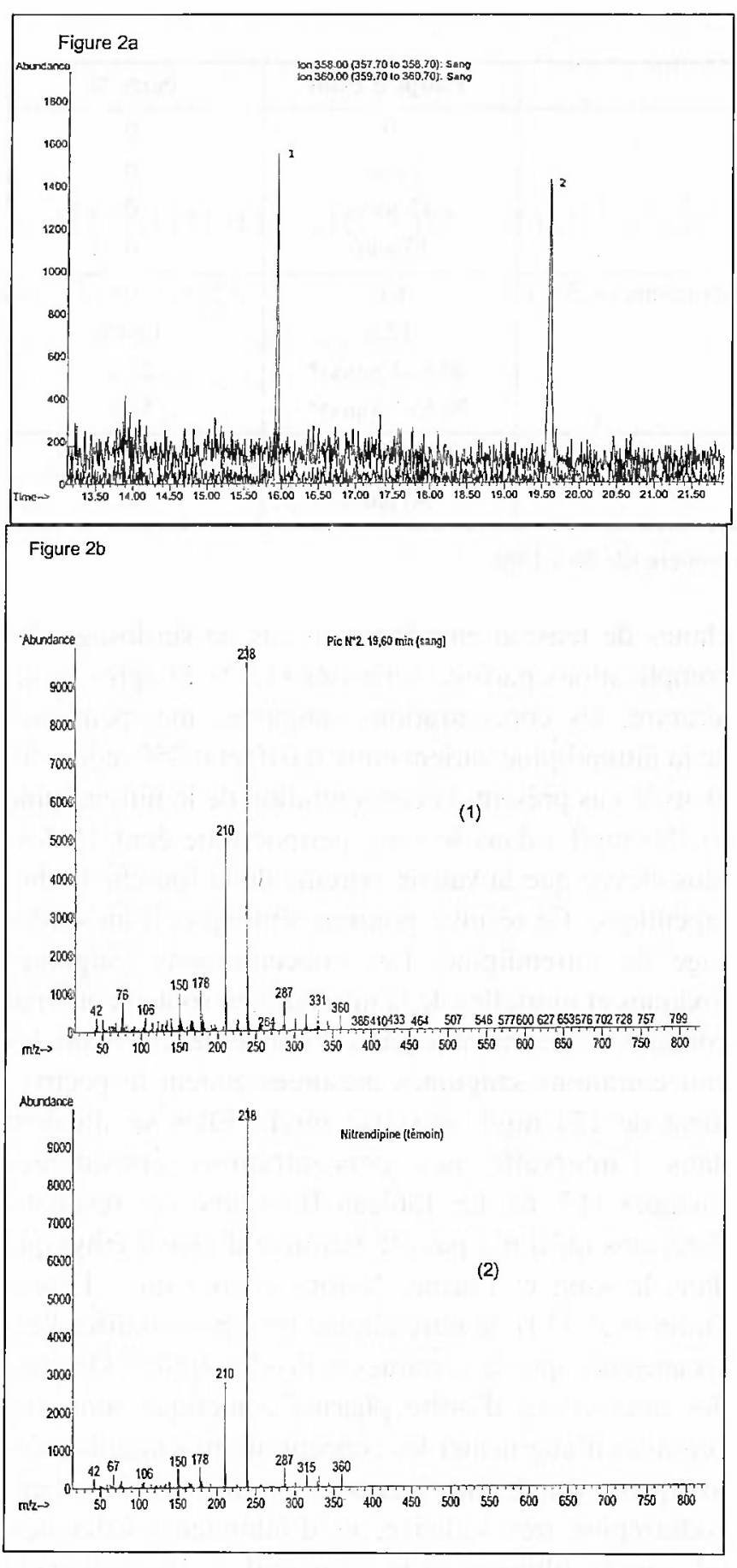

\section{Conclusion}

Les techniques mises en place (CPG-SM et CLHPDBD) sont particulièrement adaptées au screening toxicologique et au dosage de la nitrendipine. La méthode mise au point pour le dosage de la nitrendipine dans le sang par CLHP-DBD se caractérise par sa simplicité et sa fiabilité. Il n'existe aucune interférence entre la nitrendipine et la déshydronitrendipine, son produit de transformation in vitro et in vivo. La concentration déterminée dans le sang est donc attribuée au seul composé actif, la nitrendipine; cette concentration est 15 fois plus élevée que la valeur extrême de la four-

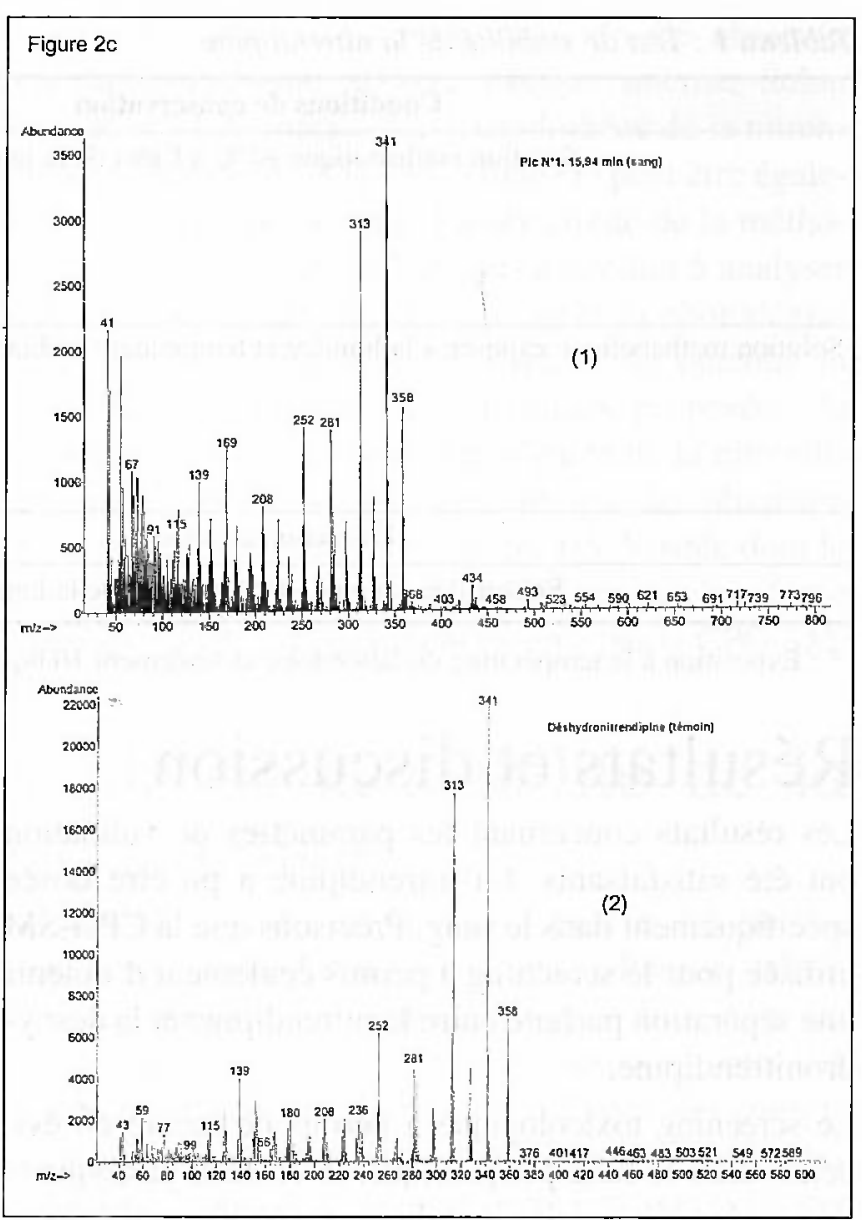

Figure 2 : Analyse du sang par CPG-SM:

a) Chronatogramme d'ions parents de la nitrendipine $\mathrm{m} / \mathrm{z}$ 360 et de la déshydronitrendipine m/z $358: I=$ déshydronitrendipine $(15,94 \mathrm{~min}) ; 2=$ nitrendipine $(19,60 \mathrm{~min})$.

b) Spectres de masse de la nitrendipine (ion parent : $\mathrm{m} / \mathrm{z}$ 360; ions fils: $\mathrm{m} / \mathrm{z} 238,210): 1=$ nitrendipine dans le sang $; 2$ = nitrendipine témoin.

c) Spectres de masse de la déshydronitrendipine (ion. parent : $\mathrm{m} / \mathrm{z} 358$; ions fils : $\mathrm{m} / \mathrm{z} 341,313): 1=$ déshydronitrendipine dans le sang ; 2 = déshydronitrendipine témoin (obtenue par photodégradation).

chette thérapeutique. Ce résultat nous conduit à envisager deux hypothèses quant à l'origine de cette concentration importante : soit la concentration en nitrendipine reflète un surdosage, soit elle est issue de l'accumulation de cette substance dans l'organisme, en raison des interactions pharmacocinétiques induites par la présence du paracétamol à concentration toxique. La présente étude, mettant en évidence la nitrendipine, le paracétamol et le triazolam à des concentrations sanguines respectives de $0,750 \mathrm{mg} / \mathrm{L}, 124 \mathrm{mg} / \mathrm{L}$ et $0,047 \mathrm{mg} / \mathrm{L}$, constitue le premier cas mortel documenté d'intoxication polymédicamenteuse où la nitrendipine est impliquée. 


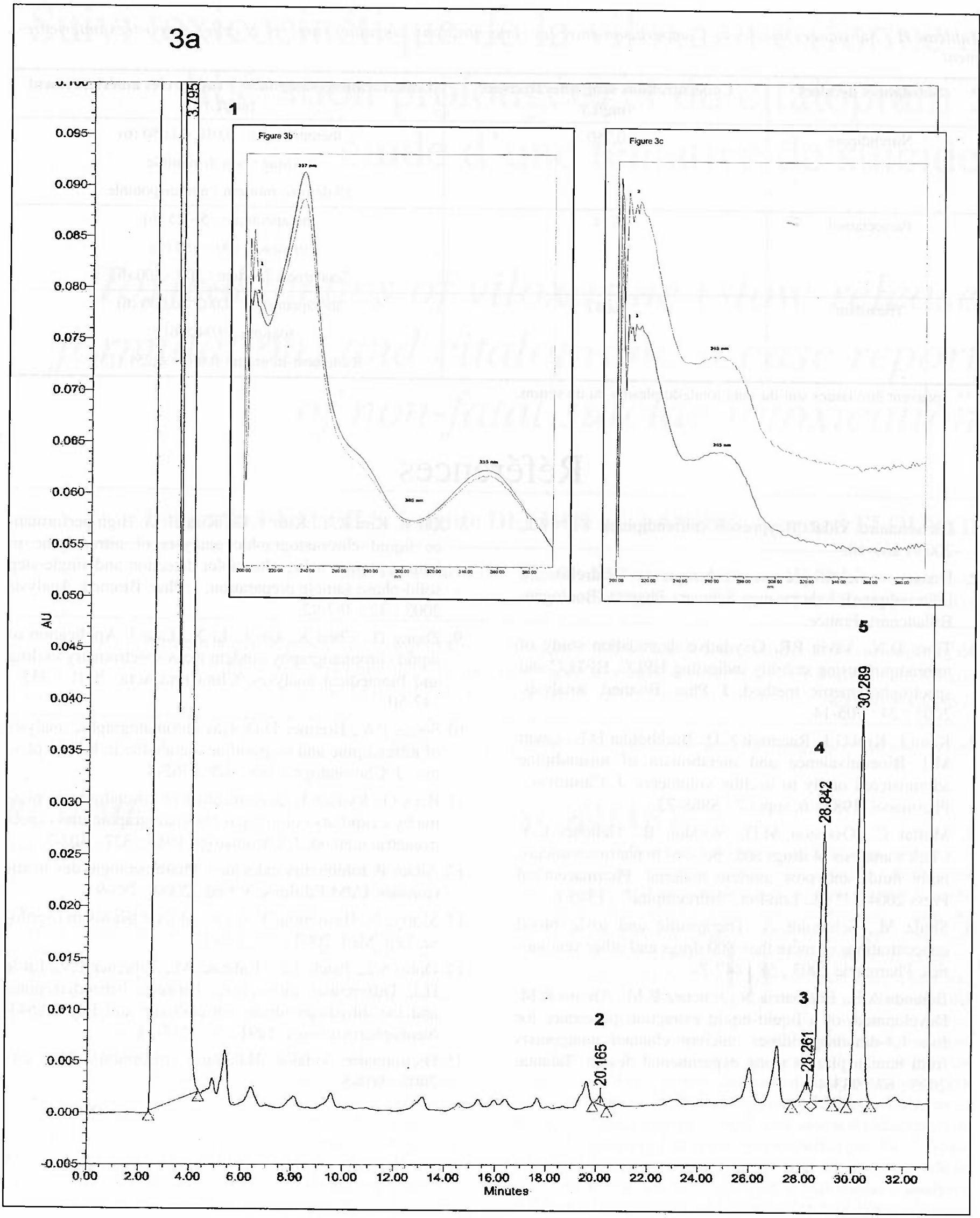

Figure 3 : Analyse du sang par CLHP-DBD :

a) Chromatogramme à $237 \mathrm{~nm}: 1=$ paracétamol $(3,79 \mathrm{~min}), 2=$ triazolam $(20,16 \mathrm{~min}), 3=$ déshydronitrendipine $(28,26$ min $)$, $4=$ nitrendipine $(28,84 \mathrm{~min})$ et $5=$ clotiazépam, étalon interne $(30,29 \mathrm{~min})$.

b) Spectre UV-Visible de la nitrendipine établi entre 200 et $400 \mathrm{~nm}: 1=$ nitrendipine dans le sang; $2=$ nitrendipine témoin. c) Spectre UV-Visible de la déshydronitrendipine établi entre 200 et $400 \mathrm{~nm}: I=$ déshydronitrendipine dans le sang ; $2=$ déshydronitrendipine témoin obtenue par photodégradation. 
Tableau II : Substances décelées - Comparaison entre les concentrations sanguines trouvées et celles rapportées antérieurement.

\begin{tabular}{|c|c|c|}
\hline $\begin{array}{c}\text { Substances décelées } \\
\text { Nitrendipine }\end{array}$ & $\begin{array}{c}\text { Concentrations sanguines trouvées } \\
(\mathbf{m g} / \mathbf{L})\end{array}$ & $\begin{array}{c}\text { Concentrations sanguines } \\
(\mathbf{m g} / \mathbf{L})\end{array}$ \\
\hline & 0,750 & $\begin{array}{c}\text { rapportées antérieurement } \\
\text { thérapeutique }: 0,010-0,050(6) \\
\text { toxique }: \text { non disponible } \\
\text { létale/post-mortem }: \text { non disponible }\end{array}$ \\
\hline Paracétamol & 124 & $\begin{array}{c}\text { thérapeutique }: 5-25(6) \\
\text { toxique }: 100-150(6) \\
\text { létale/post-mortem }: 200-300(6)\end{array}$ \\
\hline Triazolam & 0,047 & thérapeutique $: 0,002-0,020(6)$ \\
& & toxique $: 0,040(6)$ \\
& & létale/post-mortem $: 0,062-0,251(13)$
\end{tabular}

**: peuvent être issues soit du sang total, du plasma ou du sérum.

\section{Références}

1. Dictionnaire Vidal. Baypress(B) (nitrendipine), $81^{\mathrm{ims}}$ éd., $2005: 209-10$.

2. Dossier scientifique et technique : Nidrel® 20 (Nitrendipine), Laboratoires Schwarz Pharma, BoulogneBillancourt, France.

3. Tipre D.N., Vavia P.R. Oxydative degradation study of nitrendipine using stability indicating HPLC, HPTLC and spectrophotometric method. J. Phar. Biomed. Analysis. $2001 ; 24: 705-14$.

4. Kann J., Krol G.J., Raemsch K.D., Burkholder D.E., Levitt M.J. Bioequivalence and metabolism of nitrendipine administered orally to healthy volunteers. J. Cardiovasc. Pharmacol. $1984 ; 6$, suppl.7 : S968-73.

5. Moffat C., Osselton M.D., Widdop B., Galichet L.Y. Clark's analysis of drugs and poisons in pharmaceuticals, body fluids and post mortem material. Pharmaceutical Press 2004 ; 3"l ed., London. "Nitrendipine" : 1345-6.

6. Shulz M., Schmoldt A. Therapeutic and toxic blood concentrations of more than 800 drugs and other xenobiotics. Pharmazie $2003 ; 58: 447-74$.

7. Baranda A.B., Etxebarria N., Jimenez R.M., Alonso R.M. Development of a liquid-liquid extraction procedure for five 1,4-dihydropyridines calcium channel antagonists from human plasma using experimental design. Talanta. $2005 ; 67: 933-41$.
8. Oh Y., Kin K.Y., Kim Y. G., Kim H. G. High performance liquid chromatographic analysis of nitrendipine in human plasma using ultraviolet detection and single-step solid-phase sample preparation. J. Phar. Biomed. Analysis $2003 ; 32: 387-92$.

9. Zhong D., Chen X., Gu J., Li X., Guo J. Application of liquid chromatography-tanden mass spectrometry in drug and biomedical analyses. Clin.Chim.Acta. 2001 ; 313 : $147-50$.

10. Soons P.A., Breimer D.D. Gas chromatographic analysis of nitrendipine and its pyridine metabolite in human plasma . J. Chromatogr. 1988 ; $428: 362-8$.

11. Beck O., Ryman T. Quantification of nitrendipine in plasma by a capillary column gas chromatographic-mass spectrometric method. J. Chromatogr. 1985 ; 337 : 402-7.

12. Allain P. Inhibiteurs calciques. Pharmacologie des médicaments. CdM Editions, $3^{\text {ine }}$ éd., $2000 ; 288-91$.

13. Moriya F., Hashimoto $Y$. A case of fatal triazolam overdose. Leg: Med. 2003 ; 27 : 110-12.

14. Dolin S.J., Patch T.L., Rabbani M., Taberner P.V., Little H.J. Differential interactions between benzodiazepines and the dihydropyridines, nitrendipine and Bay K8644. Neuropharmacology. $1991 ; 30: 217-24$.

15. Dictionnaire Vidal@. Halcion(10 (triazolam), $81^{\text {ime }}$ éd., 2005: 904-5. 